\title{
Orobanchaceae endémicas del Perú
}

\section{Blanca León ${ }^{1,2}$}

${ }^{1}$ Museo de Historia Natural, Av. Arenales 1256, Aptdo. 14-0434, Lima 14, Perú

2 Plant Resources Center, University of Texas at Austin, Austin TX 78712 EE.UU.

blanca.leon@mail.utexas.edu

\section{Resumen}

La familia Orobanchaceae es reconocida en el Perú por presentar siete géneros y 48 especies (Brako \& Zarucchi, 1993; Stevens, 2001; Ulloa Ulloa et al., 2004), principalmente hierbas parásitas. En este trabajo reconocemos 20 taxones endémicos en Bartsia y Castilleja. Los taxones endémicos se encuentran principalmente en las regiones Mesoandina y Páramo, entre los 2150 y $4600 \mathrm{~m}$ de altitud. Once taxones se encuentran representados dentro del Sistema Nacional de Áreas Naturales Protegidas por el Estado.

Palabras claves: Orobanchaceae, Bartsia, Castilleja, Perú, endemismo, plantas endémicas.

\section{Abstract}

The Orobanchaceae are represented in Peru by seven genera and 48 species (Brako \& Zarucchi, 1993; Stevens, 2001; Ulloa Ulloa et al., 2004), mostly parasitic herbs. Here we recognize 20 endemic taxa in Bartsia and Castilleja. These endemic taxa are found mainly in Mesoandean and Paramo regions, between 2150 and 4600 m elevation. Eleven endemic taxa have been recorded in the Peruvian System of Protected Natural Areas.

Keywords: Orobanchaceae, Bartsia, Castilleja, Peru, endemism, endemic plants.

\section{Bartsia aprica Diels}

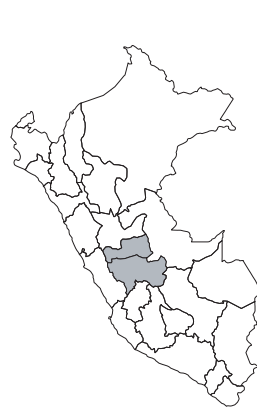

\section{VU, B1ab(iii)}

Publicación: Bot. Jahrb. Syst. 37: 430. 1906.

Colección tipo: P.C. Hutchison 640

Herbarios: UC.

Nombre común: Desconocido.

Registro departamental: JU, PA.

Regiones Ecológicas: MA, PSH; 3000$4150 \mathrm{~m}$.

SINANPE: Sin registro

Herbarios peruanos: Ninguno.

Observaciones: Hierba probablemente anual, conocida de sitios abiertos y ambientes de puna en el centro del país (Molau, 1990). Fue listada para Huánuco, pero el ejemplar que se atribuye a ese Departamento, en realidad corresponde a Pasco. Al igual que otras especies de los ambientes de puna y sitios abiertos está sujeta a modificaciones del hábitat por pastoreo y remoción de suelos.

\section{Bartsia canescens Wedd.}

\section{LC}

Publicación: Chlor. Andina 2: 123. 1860. Colección tipo: J. Dombey s.n.

Herbarios: P.

Nombre común: Desconocido.

Registro departamental: AN, AP, AY, CU, HU, HV, JU, LI, LL.

Regiones Ecológicas: MA, PSH, AA; 2150—4500 m.

SINANPE: PNH

Herbarios peruanos: CPUN (1), HAO (1), USM (2).

Observaciones: Hierba perenne, conocida de varias localidades en la vertiente del Pacífico. El ejemplar tipo fue recolectado por la expedición de Ruiz y Pavón, a fines del siglo XVIII, probablemente en la provincia de Oyón en Lima. Esta especie tiene un rango altitudinal amplio y hay registros botánicos continuos en los herbarios.

\section{Bartsia crisafullii N. Holmgr. subsp. acutiloba Molau}

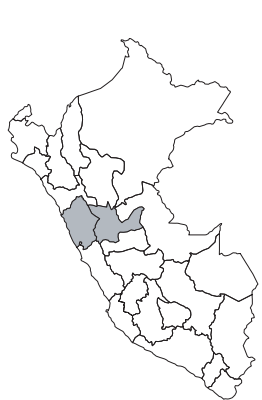

$$
\text { VU, B1a }
$$

Publicación: Opera Bot. 102: 46. 1990. Colección tipo: J.F. Macbride \& W. Featherstone 1495

Herbarios: F, PH.

Nombre común: Desconocido.

Registro departamental: AN, HU.

Regiones Ecológicas: MA; 2450—3900 m.

SINANPE: Sin registro.

Herbarios peruanos: Ninguno.

Observaciones: Este taxón anual se conoce de cinco localidades, en los valles interandinos de las Provincias de Huari en Ancash y de Ambo y Pachitea en Huánuco. Al igual que otros taxones en este género, podrían haber ejemplares de herbario en las instituciones peruanas que representen otras poblaciones, pero que no están identificadas.

\section{Bartsia elachophylla Diels}

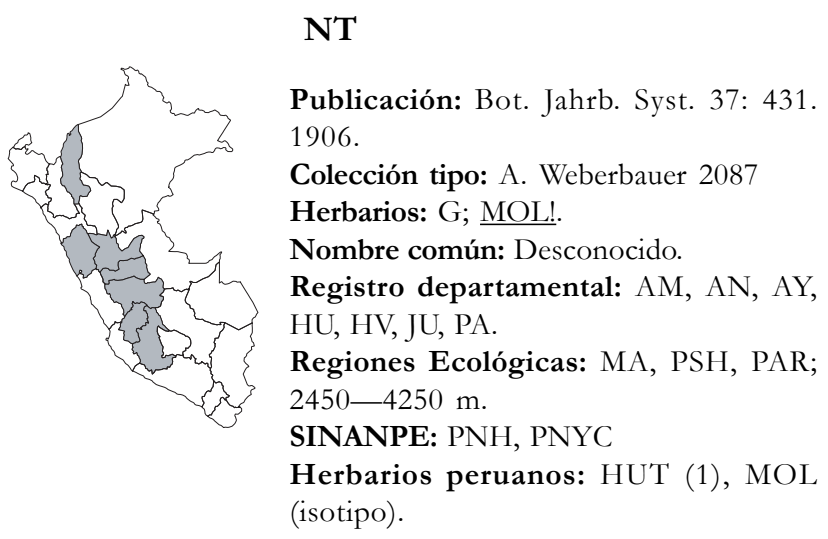

Observaciones: Hierba anual que logra alcanzar hasta $1 \mathrm{~m}$ de alto, conocida de más de diez localidades. La mayoría de las poblaciones conocidas están ubicadas en la cuenca del Santa y en los pajonales húmedos en la vertiente oriental. Molau (1990) mencionó que solamente una población está ubicada en la vertiente occidental. Por ello es probable que esta especie tenga otras poblaciones en lugares menos herborizados de los Andes. 


\section{Bartsia flava Molau subsp. minor Molau}

EN, B1ab(iii)

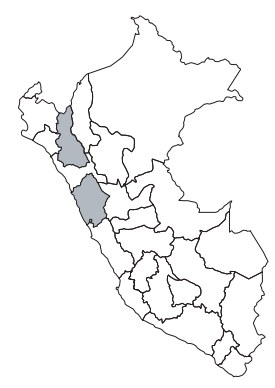

Publicación: Opera Bot. 102: 62. 1990. Colección tipo: U. Molau \& B. Eriksen 3337

Herbarios: AAU, GB; $\underline{\text { CPUN. }}$

Nombre común: Desconocido.

Registro departamental: AN, CA.

Regiones Ecológicas: PSH, PAR; 3000$3600 \mathrm{~m}$.

SINANPE: PNH

Herbarios peruanos: CPUN (isotipo), USM (5).

Observaciones: Esta subespecie no fue señalada como endémica en Brako \& Zarucchi (1993); erróneamente se listó como tal la subespecie típica. Es una hierba terrestre de ambientes paramunos y de puna. Este taxón se conoce de tres poblaciones, en un área muy reducida y fragmentada en Cajamarca y Ancash.

\section{Bartsia inaequalis Benth. subsp. duripilis (Edwin) Molau}

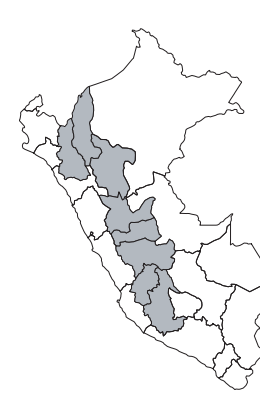

\section{LC}

Publicación: Opera Bot. 102: 54—55, f. 15H. 1990.

Colección tipo: A. Weberbauer 7553

Herbarios: F, NY, U.

Nombre común: Desconocido.

Registro departamental: AM, AY, HU, HV, JU, PA, SM.

Regiones Ecológicas: MA, BMHM; 2800-3300 m.

SINANPE: PNRA

Herbarios peruanos: USM (2).

Observaciones: Arbusto conocido de más de 16 poblaciones fragmentadas y dispersas, casi a lo largo de las vertientes orientales. Es probable que su rango de distribución sea mayor, como señaló Molau (1990) las poblaciones conocidas sólo indican la necesidad de mayor herborización en la zona montana oriental.

\section{Bartsia integrifolia Wedd.}

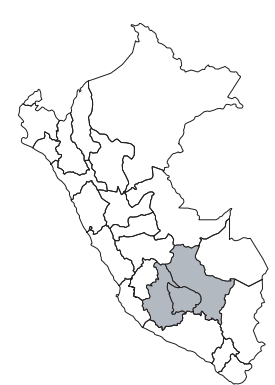

\section{VU, B1a}

Publicación: Chlor. Andina 2: 131. 1860.

Colección tipo: F. Castelnau s.n.

Herbarios: $\mathrm{P}, \mathrm{PH}$.

Nombre común: Desconocido.

Registro departamental: AP, AY, CU.

Regiones Ecológicas: MA; 2700—3500 m.

SINANPE: Sin registro.

Herbarios peruanos: Ninguno.

Observaciones: Subarbusto de ambientes mesoandinos, restringido a cuatro localidades en el centro sur del país. La localidad original se atribuye dudosamente a Lima (Molau, 1990). Aunque es una especie de fácil reconocimiento, aparentemente, no ha vuelto a ser recolectada desde 1939. Probablemente la escasez de la representación en los herbarios refleje la falta de herborización en esa parte del país. Las localidades conocidas se encuentran en tributarios de las cuencas del Mantaro, Pampas y Apurímac. Amenazas potenciales a las poblaciones de esta especie podrían estar asociadas a los incendios intencionales en matorrales.

\section{Bartsia pyricarpa Molau}

LC

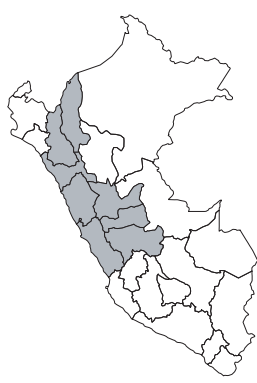

Publicación: Opera Bot. 102: 44. 1990.

Colección tipo: U. Molau et al. 1681

Herbarios: GB; CPUN!, HUT!

Nombre común: Desconocido.

Registro departamental: AM, AN, CA, HU, JU, LL, LI, PA.

Regiones Ecológicas: MA, PSH, PAR, AA; $2400-4800 \mathrm{~m}$.

SINANPE: PNH, RNJ

Herbarios peruanos: CPUN (isotipo+5), HAO (1), HUT (isotipo).

Observaciones: Hierba anual, localmente rara (Molau, 1990), pero conocida de numerosas localidades altoandinas y de valles interandinos. Habita ambientes abiertos en pajonales. Incendios intencionales podrían afectarla.

\section{Bartsia rigida Molau}

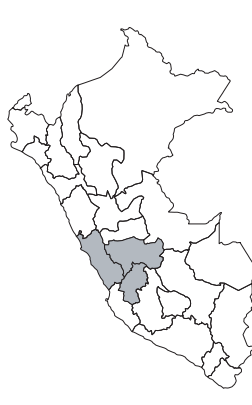

\section{NT}

Publicación: Opera Bot. 102: 78. 1990.

Colección tipo: J.F. Macbride \& W. Featherstone 735

Herbarios: F, PH.

Nombre común: Desconocido.

Registro departamental: HV, JU, LI.

Regiones Ecológicas: MA, PSH, AA; 3200-5000 m.

SINANPE: Sin registro.

Herbarios peruanos: USM (5)

Observaciones: Hierba perenne, conocida de unas 15 localidades, en el centro del país, en las cuencas del Mantaro y Rímac. La mayoría de las poblaciones proceden de la cuenca del Rímac, una de las más afectadas por contaminación y modificación del paisaje. Por su rango altitudinal, amenazas potenciales a sus poblaciones probablemente ocurran por modificación de su hábitat.

\section{Bartsia sericea Molau}

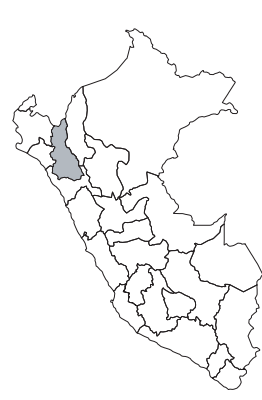

\section{VU, B1ab(iii)}

Publicación: Opera Bot. 102: 33, f. 9J-P. 1990.

Colección tipo: U. Molau et al. 1682

Herbarios: GB; CPUN!, HUT!

Nombre común: Desconocido.

Registro departamental: CA.

Regiones Ecológicas: PAR; 3400—3900 $\mathrm{m}$.

SINANPE: Sin registro.

Herbarios peruanos: CPUN (isotipo+3), HUT (isotipo+1).

Observaciones: Hierba perenne, conocida de unas cinco localidades aisladas en las Provincias vecinas de Cajamarca, Hualgayoc y Celendín, en el sur del departamento de Cajamarca. El área de presencia alcanza unos $3500 \mathrm{~km}^{2}$, hallándose en pajonales sobre laderas rocosas. 


\section{Bartsia strigosa Molau}

\section{VU, B1a}

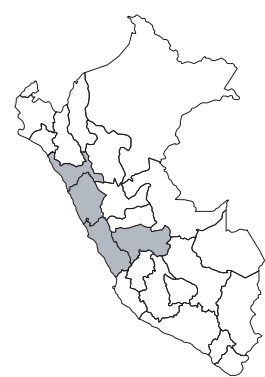

Publicación: Opera Bot. 102: 79, f. 19. 1990.

Colección tipo: E.P. Killip \& A.C. Smith 21608

Herbarios: F, NY, PH, US.

Nombre común: Desconocido.

Registro departamental: AN, JU, LI, LL. Regiones Ecológicas: MA, PAR; 3000$4100 \mathrm{~m}$.

SINANPE: PNH

Herbarios peruanos: HAO (1).

Observaciones: Subarbusto o arbusto conocido de siete localidades, ubicadas en cuencas que drenan al Pacífico, como el Santa, Chillón y Rímac. Las subpoblaciones en la cuenca del Rímac han sido herborizadas con cierta frecuencia temporal, pero no existe material de herbario reciente.

\section{Bartsia tenuis Molau}

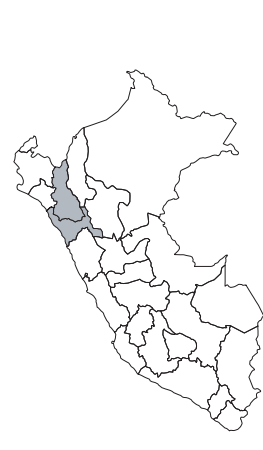

\section{VU, B1ab(iii)}

Publicación: Opera Bot. 102: 89—90, f. 25B. 1990.

Colección tipo: U. Molau et al. 1658

Herbarios: GB; CPUN!, HUT!.

Nombre común: Desconocido.

Registro departamental: CA, LL.

Regiones Ecológicas: PAR; 2800—4200

$\mathrm{m}$.

SINANPE: Sin registro.

Herbarios peruanos: CPUN (isotipo+4), HAO (1), HUT (isotipo), USM (2).

Observaciones: Hierba perenne, conocida de 10 localidades en el sur de Cajamarca y centro de La Libertad. El hábitat en la localidad original, así como en los alrededores de la ciudad de Cajamarca, está deteriorado por pastoreo intensivo. Su amplitud altitudinal y la presencia en sitios rocosos puede aminorar esta amenaza.

\section{Bartsia tomentosa Molau}

\section{LC}

Publicación: Opera Bot. 102: 55-56, f. 15K-Q. 1990.

Colección tipo: U. Molau et al. 1680

Herbarios: GB; CPUN!, HUT!

Nombre común: Desconocido.

Registro departamental: AN, CA, LL, PA. Regiones Ecológicas: PSH, PAR; 3450$4600 \mathrm{~m}$.

SINANPE: PNH

Herbarios peruanos: CPUN (isotipo+5), HUT (isotipo+4).

Observaciones: Hierba perenne conocida de varias localidades, en cuencas de ríos que drenan al Pacífico, con la excepción de la población en Pasco. En Ancash, se ha registrado tanto en la Cordillera Blanca como en la Negra. Probablemente su distribución sea más amplia que la obtenida de los datos de herbarios y literatura.

\section{Bartsia tricolor Molau}

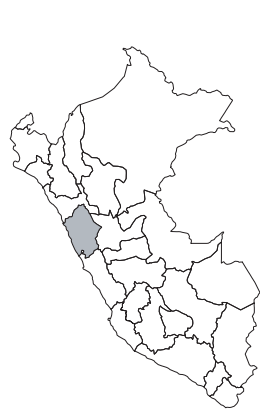

VU, B1a

Publicación: Opera Bot. 102: 79—80, f. 19, 22L-O. 1990.

Colección tipo: U. Molau et al. 538

Herbarios: GB, S; USM.

Nombre común: Desconocido.

Registro departamental: AN.

Regiones Ecológicas: PSH, AA; 3875$4700 \mathrm{~m}$.

SINANPE: PNH

Herbarios peruanos: CPUN (3), HUT (8), USM (isotipo+1).

Observaciones: Hierba cespitosa, conocida de unas diez localidades, la mayoría en la cuenca del Santa. Esta especie ocupa tanto la Cordillera Blanca como la Negra; en esta última se conoce también de la vertiente occidental.

\section{Castilleja alpicola T.I. Chuang \& Heckard}

\section{DD}

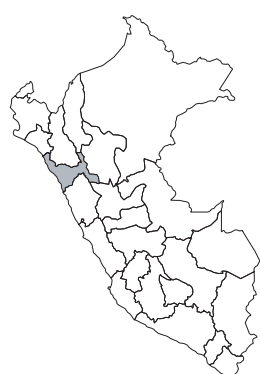

Publicación: Syst. Bot. 17(3): 420, f. 2DF, 3. 1992.

Colección tipo: A. Sagástegui A. et al. 11969

Herbarios: GB; $\underline{\text { HUT. }}$.

Nombre común: Desconocido.

Registro departamental: LL.

Regiones Ecológicas: PAR; $4000 \mathrm{~m}$.

SINANPE: Sin registro.

Herbarios peruanos: HUT (isotipo).

Observaciones: Herba probablemente anual, conocida de una localidad, en la vertiente occidental de La Libertad. Aparentemente, no ha vuelto a ser recolectada desde 1984, debido probablemente a lo poco herborizado de la zona. $\mathrm{Al}$ igual que otras especies de la jalca, estudios en el futuro podrán contribuir a aclarar la biología y el estado de conservación de esta especie.

\section{Castilleja peruviana Chuang \& Heckard}

\section{NT}

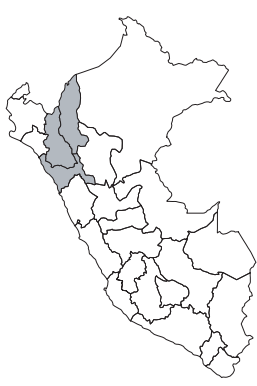

Publicación: Syst. Bot. 17(3): 426—427, f. 6A-C. 1992.

Colección tipo: T. Duncan 2846

Herbarios: F, GH, K, MO, NY, UC, US; USM.

Nombre común: Desconocido.

Registro departamental: AM, CA, LL. Regiones Ecológicas: MA, PAR; 2500_ $3500 \mathrm{~m}$.

SINANPE: Sin registro.

Herbarios peruanos: CPUN (1), HUT (1), USM (isotipo citado).

Observaciones: Hierba anual conocida de diez poblaciones fragmentadas, en ambas vertientes andinas. Habita ambientes rocosos, con vegetación herbácea. 


\section{Castilleja profunda Chuang \& Heckard}

\section{NT}

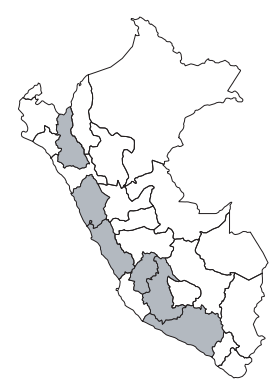

Publicación: Syst. Bot. 17(3): 427, 429, f. 4D-F. 1992.

Colección tipo: A.S. Hitchcock 22404

Herbarios: US.

Nombre común: Desconocido.

Registro departamental: AN, AR, AY, CA, HV, LI.

Regiones Ecológicas: DST, MDE, MA; 100-3500 m.

SINANPE: PNH.

Herbarios peruanos: USM?

Observaciones: Hierba anual conocida de más de diez localidades dispersas, tanto en las cuencas del Santa, Marañón y Mantaro, como en ambientes de lomas de la costa de Arequipa. No fue reconocida como un endemismo por Brako \& Zarucchi (1993), pero aquí se la acepta como tal. Probablemente su distribución sea mayor que la conocida, pues presenta una amplitud ecológica grande.

\section{Castilleja pseudopallescens Edwin}

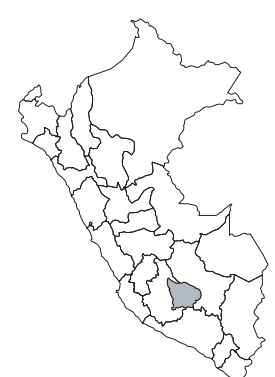

\section{DD}

Publicación: Phytologia 19(6): 404. 1970. Colección tipo: C. Vargas C. 5800

Herbarios: F.

Nombre común: Desconocido.

Registro departamental: AP.

Regiones Ecológicas: MA; $2700 \mathrm{~m}$.

SINANPE: Sin registro.

Herbarios peruanos: Ninguno.

Observaciones: Hierba perenne conocida solamente de una localidad, en la cuenca del Vilcabamba, un afluente del Apurímac. Esta localidad ha sido escasamente herborizada y esto contribuya tal vez a lo poco que se sabe de esta especie.

\section{Castilleja vadosa Chuang \& Heckard}

\section{LC}

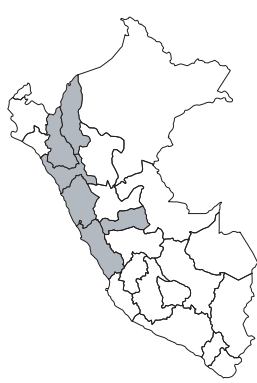

Publicación: Syst. Bot. 17(3): 429—430, f. 6 D-H. 1992.

Colección tipo: P.C. Hutchison et al. 6266 Herbarios: F, K, MO, NY, UC, US; USM. Nombre común: Desconocido.

Registro departamental: AM, AN, CA, LL, LI, PA.

Regiones Ecológicas: MA, PSH, PAR; 2500-4050 m.

SINANPE: PNH

Herbarios peruanos: USM (isotipo citado).

Observaciones: Hierba anual cercanamente vinculada a otra endémica Castilleja perwivana, con la que comparte el mismo hábitat en su rango de distribución. Se conoce de más de diez localidades en ambas vertientes andinas.

\section{Castilleja virgatoides Edwin}

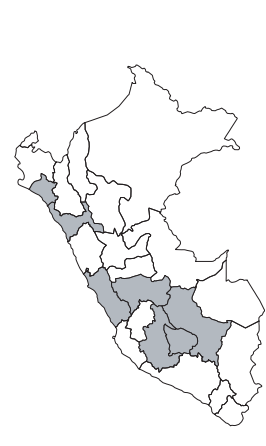

NT

Publicación: Phytologia 19(6): 405-406. 1970.

Colección tipo: F.W. Pennell 13699

Herbarios: F, GH, NY, PH.

Nombre común: Desconocido.

Registro departamental: AP, AY, CU, JU, LA, LL, LI.

Regiones Ecológicas: MA, PSH, AA; 2700-4600 m.

SINANPE: SNA

Herbarios peruanos: Ninguno.

Observaciones: Hierba perenne conocida de varias localidades dispersas en el norte y centro del país, en ambas vertientes andinas, en las cuencas del Chusgón, Rímac, Urubamba, entre otras. Probablemente amenazas a sus poblaciones estén asociadas a incendios intencionales. 